\title{
Nanostructure and compositional segregation of soft magnetic FeNi-based nanocomposites with multiple nanocrystalline phases
}

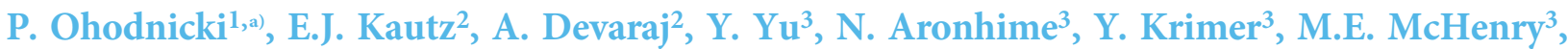 \\ A. Leary ${ }^{4}$ \\ ${ }^{1}$ Mechanical Engineering and Materials Science, University of Pittsburgh, Pittsburgh, Pennsylvania 15261, USA \\ ${ }^{2}$ Pacific Northwest National Laboratory, Richland, Washington 99354, USA \\ ${ }^{3}$ Materials Science and Engineering, Carnegie Mellon University, Pittsburgh, Pennsylvania 15213, USA \\ ${ }^{4}$ Materials and Structures Division, NASA Glenn Research Center, Cleveland, OH 44135, USA

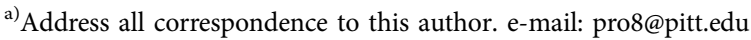

Received: 14 March 2020; accepted: 5 August 2020; published online: 4 February 2021

Soft magnetic metal amorphous nanocomposite alloys are produced through rapid solidification and thermal annealing yielding nanocrystals embedded within an amorphous precursor. Similar free energies in Co-rich and FeNi-based alloy systems result in multiple nanocrystalline phases being formed during devitrification. Studies of multi-phase crystallization processes have been reported for Co-rich alloys but relatively few have investigated FeNi-based systems. A detailed characterization of compositional partitioning and microstructure of an optimally annealed FeNi-based MANC $\left(\mathrm{Fe}_{70} \mathrm{Ni}_{30}\right)_{80} \mathrm{Nb}_{4} \mathrm{Si}_{2} \mathrm{~B}_{14}$ alloy is presented through complementary high-resolution transmission electron microscopy (HRTEM) and atom probe tomography (APT). HRTEM demonstrates orientation relationships between FCC and BCC nanocrystals, suggesting heterogeneous nucleation of nanocrystals in the amorphous matrix or a cooperative mechanism of nucleation between BCC and FCC nanocrystallites. APT results show evidence for (i) the segregation of Fe and Ni between nanocrystals of different phases, (ii) B partitioning to the amorphous phase, and (iii) an Nb-enriched shell surrounding nanocrystals.

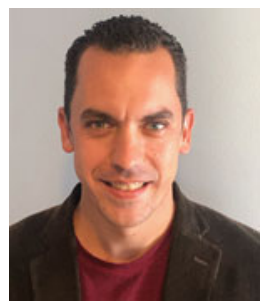

Paul R. Ohodnicki Jr. is Associate Professor of Mechanical Engineering and Materials Science at the University of Pittsburgh and Director of the Engineering Science program. Before joining the University of Pittsburgh, he was a materials scientist and technical portfolio lead in the Functional Materials Team of the Materials Engineering \& Manufacturing Directorate of the National Energy Technology Laboratory, Pittsburgh from 2011. He graduated from the University of Pittsburgh in 2005 with a B.Phil. in engineering physics and a B.A. in economics and subsequently earned his M.S. (2006) and Ph.D. (2008) in materials science and engineering from Carnegie Mellon University. From 2008 to 2010 he was a visiting research scientist at PPG Industries. During his time with the laboratory, he was responsible for overseeing projects spanning sensing and power electronics, with emphasis on advanced devices and enabling functional materials for photonic and wireless sensing as well as power magnetics component and materials design. Ohodnicki is recipient of the Federal Employee Rookie of the Year Award (2012), Presidential Early Career Award in Science and Engineering (2016), and the Advanced Manufacturing and Materials Innovation Category Award for the Carnegie Science Center (2012, 2017 and 2019). In 2017 he was a finalist for the Samuel J. Heyman Service to America Medal.

\section{Introduction}

Metal amorphous nanocomposite (MANC) alloy systems are of significant interest for a range of emerging soft magnetics applications which include, but are not limited to, inductors, transformers, and rotating electrical machinery such as motors [1]. As compared with bulk alloys such as electrical steels and permalloy, higher effective resistivity and thinner laminations permit the operation to higher switching frequencies with 
relatively low losses. When combined with higher saturation inductions attainable as compared with high-frequency ferrite core materials, these materials show ideal technical performance characteristics for medium frequency $(1-100 \mathrm{kHz})$ and medium to high power $(\mathrm{kW}+)$ applications. MANC alloy systems are typically produced through a rapid solidification process such as planar flow casting to generate an amorphous metal ribbon (AMR) followed by a thermal annealing treatment to partially devitrify the ribbon and form a stabilized nanocomposite MANC microstructure. Existing commercial alloy systems produced in bulk quantities are based upon the quaternary $\mathrm{FeNbSiBCu}$ system and contain only a single transition metal ferromagnetic element $(\mathrm{Fe})$ such that the MANC microstructure ultimately obtained is comprised of a nanocrystalline BCC-derivative $\mathrm{Fe}-\mathrm{Si} \mathrm{DO}_{3}$ phase surrounded by an amorphous matrix [2]. Newer alloy systems are being explored that have substantially modified compositions with a significant content of other ferromagnetic transition metal elements such that other close-packed nanocrystalline phases (e.g., FCC, HCP) become similar or lower in free energy as compared with BCC-derivative phases. These alloys are based upon (i) high Co-containing alloys in which nanoscale BCC, FCC, and HCP (as well as highly faulted close-packed) grains can be found in varying proportions and (ii) FeNi alloys for which nanoscale FCC grains become a significant or dominant crystalline phase after optimized annealing treatments $[3,4,5$, $6,7,8,9]$. These alloy systems are attractive for numerous applications, as they exhibit unique mechanical and magnetic properties which make them highly scalable from a manufacturing perspective and exceptionally tunable in terms of magnetic properties through the application of field and mechanical strain during thermal annealing $[4,8,10,11,12$, 13].

A significant body of work has been focused on the Co-rich alloy system such that detailed microstructural and local chemical composition analysis has been performed and thermodynamic models have been proposed to describe and rationalize phase evolution and compositional partitioning during crystallization $[14,15,16]$. More specifically, isothermal annealing experiments of CoFeZrB-based alloy systems were explored in detail across the entire range of $(\mathrm{Fe}, \mathrm{Co})$-content for fixed glass former compositions (i.e., an FeCo pseudo-binary system) illustrating a number of trends $[9,14,15,17]$ :

(1) Body-centered cubic nanocrystalline phases tend to dominate across the pseudo-binary composition until Fe: Co ratio approaching 9:1 where significant volume fractions of close-packed nanocrystalline phases are identified (FCC, HCP, and highly faulted).

(2) At lower temperatures and shorter annealing times, BCC nanocrystallites tend to form first followed by an increased volume fraction of close-packed nanocrystals at higher temperatures and longer annealing times in compositions where BCC, FCC, and HCP mixtures are observed.

(3) Compositional segregation can be observed between various nanocrystalline phases and trends could be rationalized using classical nucleation arguments and the parallel tangency condition.

Trends reported were further verified and confirmed through a detailed high-temperature X-ray diffraction study that allowed for in situ confirmation of initial BCC phase nucleation followed by close-packed phase formation at higher annealing temperatures [7]. More recent work based upon $\mathrm{CoNbSiBCu}$ alloy systems with small additions of $\mathrm{Fe}, \mathrm{Mn}$, and other transition metal elements has also demonstrated the presence of close-packed nanocrystalline phases and multiphase nanocrystalline mixtures within an amorphous matrix [13]. Mechanistic explanation remains elusive and current theories suggest enhanced ductility is predominantly associated with the intergranular amorphous phase composition [10], but enhanced ductility of Co-rich alloy systems are qualitatively correlated with a substantial volume fraction of close-packed nanocrystalline phases such that nanocrystal phase identity may also play a role.

In the case of FeNi-based alloy systems, interesting observations have also been reported in the literature regarding the interplay between BCC and FCC based nanocrystals forming during primary crystallization processes. A detailed high-temperature X-ray diffraction study of an $\left(\mathrm{Fe}_{70} \mathrm{Ni}_{30}\right)_{88} \mathrm{Zr}_{7} \mathrm{~B}_{4} \mathrm{Cu}_{1}$ alloy demonstrated initial nucleation of BCC nanocrystals during the early stages of crystallization followed by the formation of FCC nanocrystals at higher annealing temperatures [5], with retention of FCC metastable nanocrystals upon cooling to room temperature. In a detailed study of the $\left(\mathrm{Fe}_{1-x} \mathrm{Ni}_{x}\right)_{80} \mathrm{Nb}_{4} \mathrm{Si}_{2} \mathrm{~B}_{14}$ alloy system with varying $\mathrm{Fe}$ and $\mathrm{Ni}$ content, the $x=0.3$ composition was found to lie approximately at the transition between predominantly FCC as compared with BCC nanocrystals with BCC phase dominating in the more Fe-rich compositions as would be expected $[3,4]$. And as observed for the FeNiZrB-based alloys, a greater volume fraction of BCC relative to FCC nanocrystals could be observed at lower annealing temperatures. Despite these initial studies reporting the structural characterization of multi-phase crystallization processes, detailed high-resolution transmission electron microscopy (HRTEM) and compositional segregation analysis has not been reported for FeNi-based MANC alloys to date. To further understand microstructural and compositional segregation in alloys of $x=0.3$ composition for which a significant volume fraction of both phases can be found, HRTEM and atom probe tomography (APT) analysis was performed on an optimally annealed sample as described in detail in subsequent sections. 


\section{Experimental Results and Discussion}

As reported in prior studies, the samples investigated here exhibit a mixture of BCC and FCC nanocrystals within the sample bulk (greater than approximately $500 \mathrm{~nm}$ from the ribbon surfaces) after the optimized annealing treatment. A bright-field transmission electron microscopy (TEM) image is presented in Fig. 1 along with a selected area diffraction pattern, confirming the multi-phase nanocrystalline microstructure. Detailed HRTEM studies were also performed to better understand the microstructure of the multi-phase nanocomposite alloy systems. Individual nanocrystals surrounded by an intergranular amorphous phase were identified and could be indexed as either BCC or FCC phase. In Fig. 1(c), a BCC nanocrystal oriented along a [111]-type zone axis is identified with a diameter of approximately $10 \mathrm{~nm}$. In Fig. 1(d), an FCC nanocrystal oriented along a [110]-type zone axis was found with a diameter of only approximately $3 \mathrm{~nm}$.

In addition to individual nanocrystals separated by an amorphous matrix, observations were also made for regions in which BCC and FCC nanocrystals were found to be in intimate contact and displayed one of the well-known orientation relationships reported previously in the literature [18]. Several examples of these regions are presented below in Fig. 2. Such observations have also been detailed in a prior Co-rich alloy [16]. In Fig. 2(a), two individual BCC and FCC nanocrystalline grains are found to be in direct contact with one another and exhibit a Nishiyama-Wassermann orientation relationship with the BCC in a [001] zone axis and the FCC in a [0-1-1] zone axis orientation $[19,20]$. In Fig. 2(b), two individual
BCC and FCC nanocrystalline grains are found to be contact with a Kurdjumov-Sachs orientation relationship with the BCC in a [1-11] zone axis and the FCC in a [110] zone axis orientation [21]. Cube-on-cube orientation relationships have also been observed. Nanostructured BCC and FCC grains in direct contact with orientation relationships suggest heterogeneous nucleation of one phase on another and/or a cooperative nucleation process between the multiple crystalline phases.

To explore compositional segregation associated with the multi-phase nanocrystallization process, APT was performed for both as-cast and annealed samples. The average estimated composition of both as-cast and annealed samples determined via $\mathrm{APT}$ is presented in Table 1 and is in reasonable agreement with one another and generally consistent with the expected nominal alloy composition of $\left(\mathrm{Fe}_{70} \mathrm{Ni}_{30}\right)_{80} \mathrm{Nb}_{4} \mathrm{Si}_{2} \mathrm{~B}_{14}$, although estimated $\mathrm{Nb}$ content is smaller than expected by approximately $2.5-3$ at.\%.

The extent of element segregation after annealing was investigated by performing frequency distribution analysis for both as-cast and annealed conditions, with results provided in Fig. 3. Both as-cast and annealed data sets were partitioned into bins containing 200 ions (corresponding to a volume of $2.36 \mathrm{~nm}^{3}$ ), where composition in each bin was determined and plotted as a histogram. In the as-cast sample [Fig. 3(a)], the frequency distribution of $\mathrm{Fe}, \mathrm{Ni}, \mathrm{B}, \mathrm{Si}$, and $\mathrm{Nb}$ agrees well with the binomial distribution, whereas the annealed sample shows a clear deviation from random distribution [Fig. 3(b)]. Additionally, Pearson correlation coefficients $(\mu)$ were calculated to quantify the extent of deviation from binomial (a)

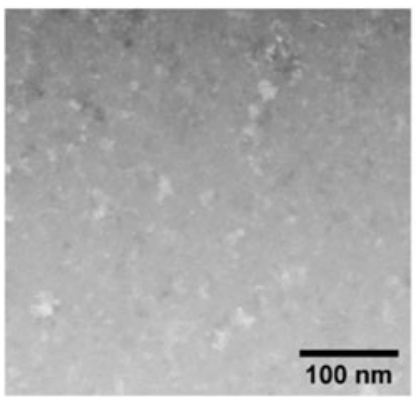

(b)

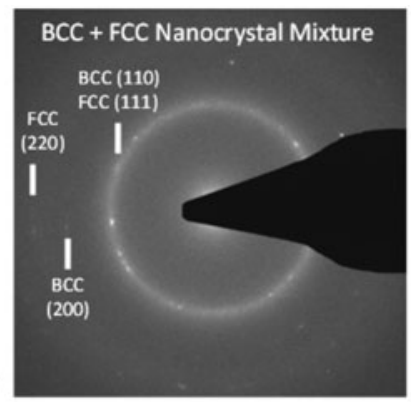

Figure 1: (a) Conventional bright-field TEM image and (b) selected area diffraction pattern confirming a nanocrystalline microstructure comprised of a mixture of $B C C$ and FCC nanocrystals within the bulk of the annealed ribbon sample. HRTEM images are also presented for regions in which (c) $\mathrm{BCC}$ and (d) $\mathrm{FCC}$ nanocrystals are identified in zone axis orientation ([111]-type and [110]-type, respectively). 
(a)
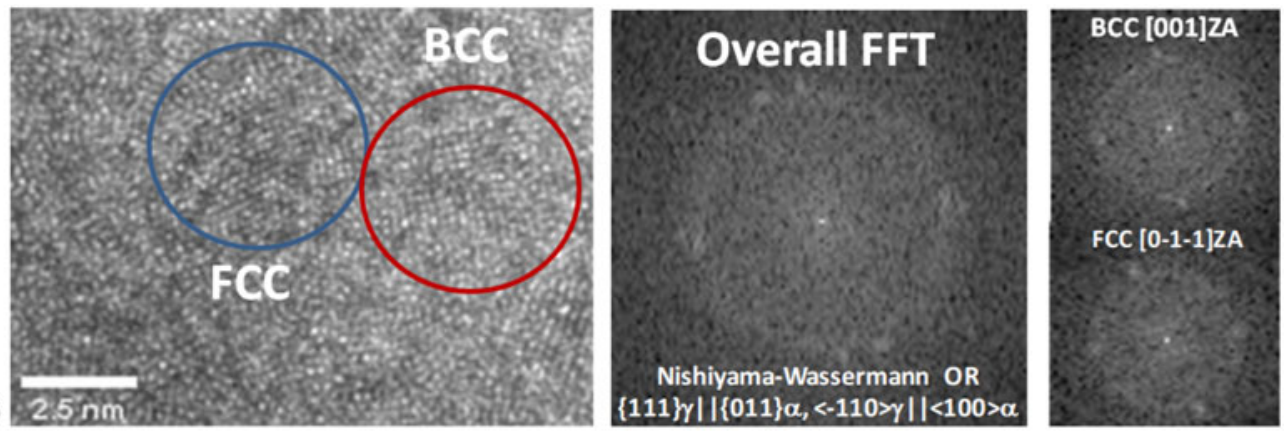

(b)
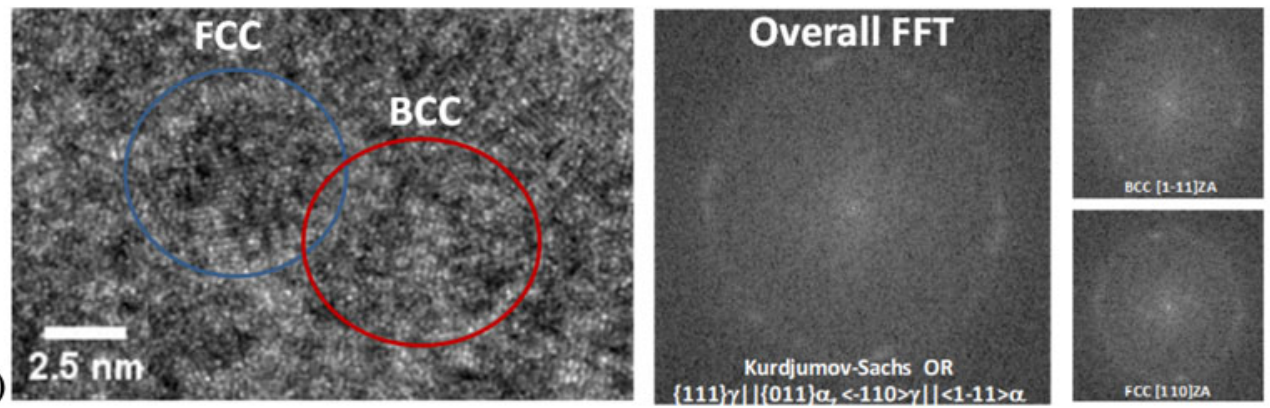

Figure 2: (a) HRTEM image and selected fast Fourier transforms showing a BCC and FCC grain on a zone axis orientation ([001] and [0-1-1], respectively) with a Nishiyama-Wassermann orientation relationship. (b) Corresponding HRTEM image and selected fast Fourier transforms showing a BCC and FCC grain on a zone axis orientation ([1-1-1] and [110], respectively) with a Kurdjumov-Sachs orientation relationship.

TABLE 1: Composition of as-cast and annealed $\left(\mathrm{Fe}_{70} \mathrm{Ni}_{30}\right)_{80} \mathrm{Nb}_{4} \mathrm{Si}_{2} \mathrm{~B}_{14}$ analyzed via APT.

\begin{tabular}{lrrrrr}
\hline \hline & \multicolumn{2}{c}{ As-Cast } & & \multicolumn{2}{c}{ Annealed } \\
\cline { 2 - 3 } \cline { 6 - 6 } & \multicolumn{1}{c}{ at.\% } & Error (\%) & & at.\% & Error (\%) \\
\hline $\mathrm{B}$ & 12.365 & 0.022 & & 12.119 & 0.023 \\
$\mathrm{Si}$ & 2.573 & 0.010 & & 2.502 & 0.010 \\
$\mathrm{Fe}$ & 53.302 & 0.053 & & 54.128 & 0.058 \\
$\mathrm{Ni}$ & 30.242 & 0.037 & & 30.102 & 0.039 \\
$\mathrm{Nb}$ & 1.450 & 0.007 & & 1.065 & 0.007 \\
\hline \hline
\end{tabular}

distribution, with results presented in Fig. 3(c) [22, 23, 24]. The Pearson correlation coefficient is a statistical metric that has a value between 0 and 1 , where 0 indicates random distribution and 1 indicates a complete association between solute atoms [22]. We find that $\mu$ is $<0.1$ for all elements in the as-cast condition, indicating near random distribution. In contrast, after annealing the $\mu$-values increased by a factor of 3-10 for all elements pointing to the increased extent of elemental segregation after annealing.

By visually analyzing 3D element distribution in APT reconstructions, three distinct regions of enrichment were identified: (i) Fe-enriched, (ii) Ni-enriched, and (iii) B-enriched regions. 3D element distribution maps shown in Figs. 4(a) and 4(b) illustrate regions in which either Fe or Ni is enriched. The $\mathrm{Fe}$ and $\mathrm{Ni}$ enriched regions are also highlighted in Figs. 4 (c) and 4(d), in which 54 at.\% $\mathrm{Fe}$ and 32 at.\% $\mathrm{Ni}$ iso- concentration surfaces are shown with corresponding 2D contour plots for $\mathrm{Fe}$ and $\mathrm{Ni}$, respectively. 2D composition contour plots were generated from a rectangular region of interest placed inside the reconstruction, with a thickness of $1 \mathrm{~nm}$ and varying height and width dimensions, where composition was analyzed along the thickness direction. Compositions across the $\mathrm{Fe}$ and $\mathrm{Ni}$ rich regions were analyzed using proximity histograms [25] across 54 at.\% $\mathrm{Fe}$ and 32 at.\% $\mathrm{Ni}$ isoconcentration surfaces and are presented in Figs. 4(e) and 4 (f), respectively. Error reported in tables and composition profiles represents point-counting error. Concentration profiles show a clear reduction in $\mathrm{B}, \mathrm{Si}$, and $\mathrm{Nb}$ content within the $\mathrm{Fe}$ and Ni-rich regions. Slight enrichment in $\mathrm{Ni}$ can also be observed at the interface of Fe-rich regions in Fig 4(e), and similarly an enrichment of $\mathrm{Fe}$ adjacent to the Ni-rich region is shown in Fig. 4(f). The composition of each $\mathrm{Fe}, \mathrm{Ni}$, and $\mathrm{B}$ enriched region was estimated using regions enclosed by a 54 at.\% $\mathrm{Fe}, 32$ at.\% Ni, and 12 at.\% B iso-concentration surfaces, with results given in Fig. 4(g). The corresponding volume fraction of each region is given in parentheses. Based on expectations for phases identified through TEM (Figs. 1 and 2) Fe-enriched regions can be identified as BCC nanocrystals, $\mathrm{Ni}$-enriched regions are identified as FCC nanocrystals, and B-enriched regions are identified as the intergranular amorphous phase.

From the Fig. 4 concentration profiles, Fe-enrichment clearly corresponds to $\mathrm{Ni}$ depletion and vice versa. Fe and $\mathrm{Ni}$ 

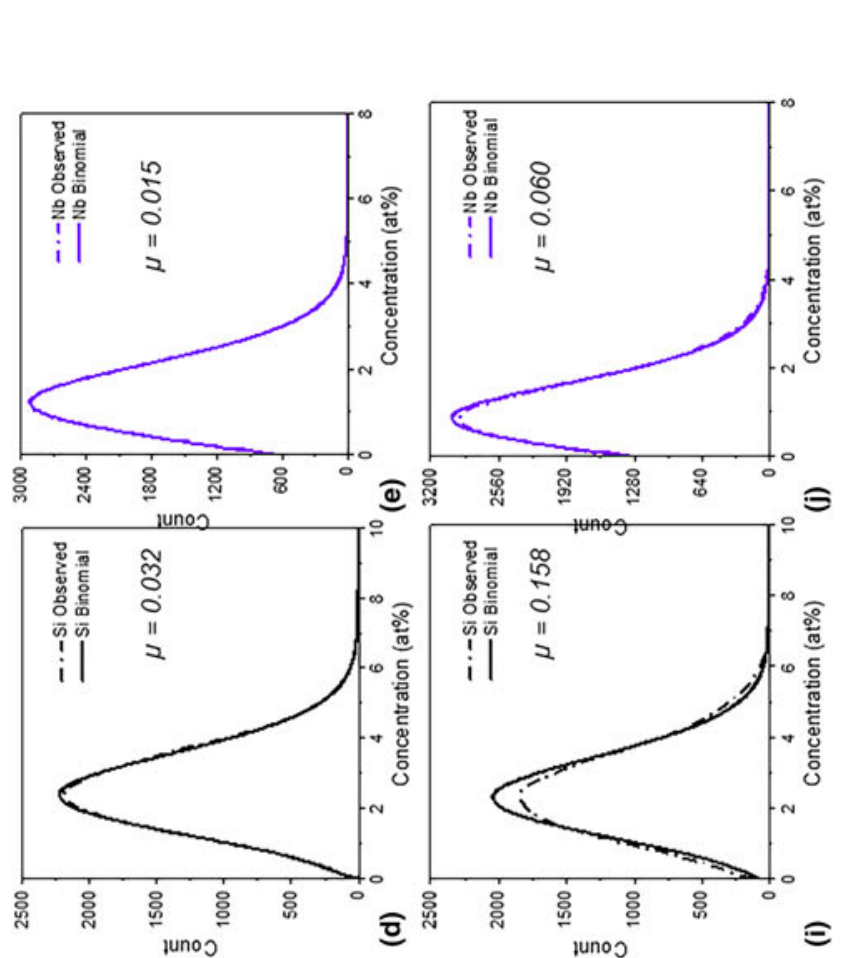

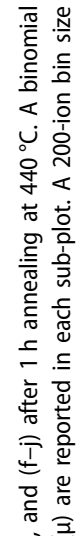

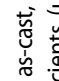

के

遂

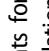

है

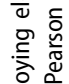
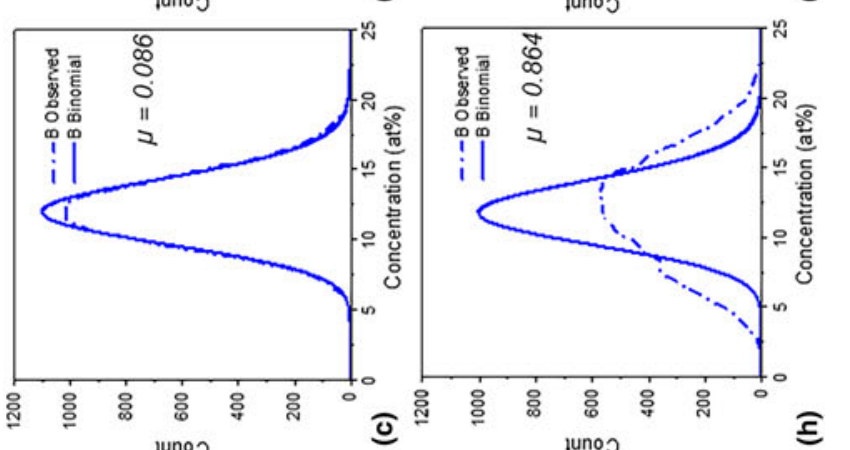

䎡 응 웡 ㅎํㅇ

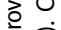
흘
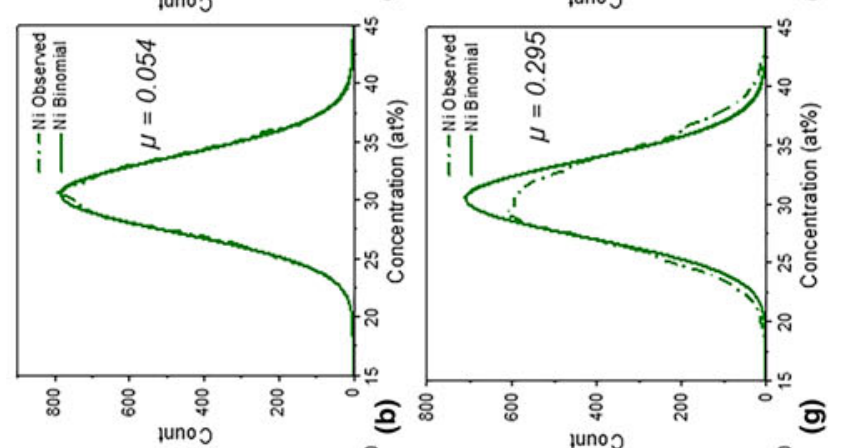

$\sum_{\infty}^{0}$

잉
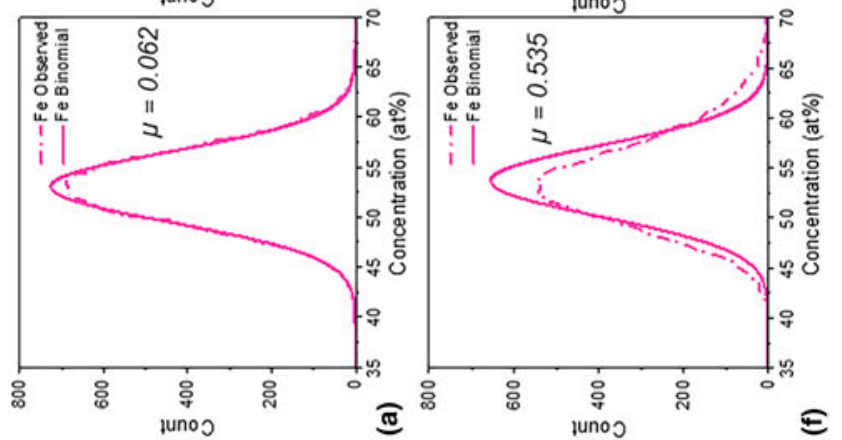

은 

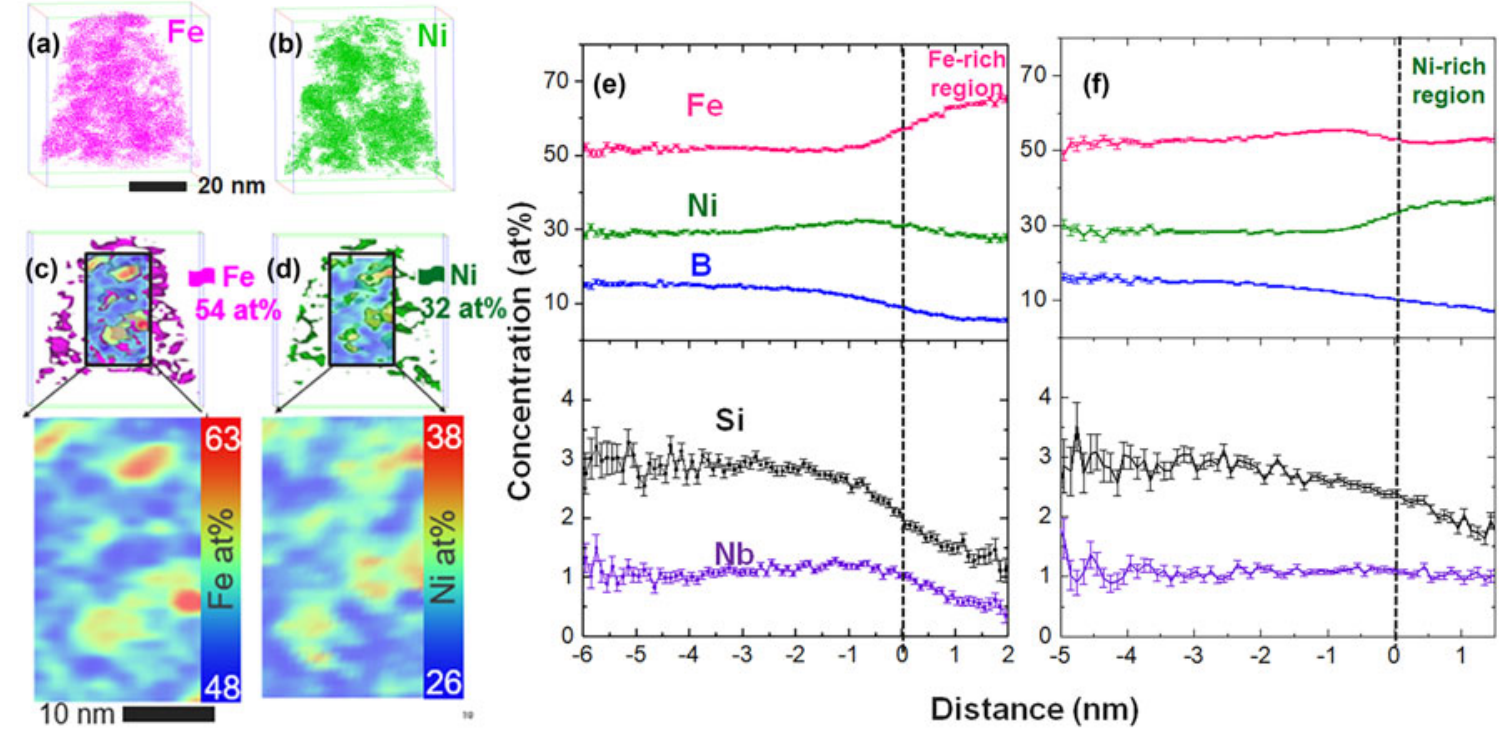

\begin{tabular}{|c|c|c|c|c|c|c|}
\hline & \multicolumn{2}{|c|}{ Fe-rich ( $35.43 \mathrm{vol} \%)$} & \multicolumn{2}{|c|}{ Ni-rich (22.45 vol \%) } & \multicolumn{2}{|c|}{ B-rich (42.12 vol\%) } \\
\hline & at $\%$ & error (\%) & at $\%$ & error $(\%)$ & at $\%$ & error (\%) \\
\hline B & 8.85 & 0.03 & 8.98 & 0.04 & 15.11 & 0.04 \\
\hline Si & 1.91 & 0.01 & 2.04 & 0.02 & 2.91 & 0.02 \\
\hline $\mathrm{Fe}$ & 58.49 & 0.10 & 52.56 & 0.12 & 52.18 & 0.09 \\
\hline $\mathrm{Ni}$ & 29.76 & 0.06 & 35.32 & 0.09 & 28.65 & 0.06 \\
\hline $\mathrm{Nb}$ & 0.93 & 0.01 & 1.04 & 0.01 & 1.07 & 0.01 \\
\hline
\end{tabular}

Figure 4: Compositional analysis of Fe-Ni-B-Si-Nb annealed at $1 \mathrm{~h}$ at $440{ }^{\circ} \mathrm{C}$ performed via APT. (a) Fe-enriched regions defined by a 54 at.\% iso-concentration surface and (b) Ni-enriched region defined by a 32 at.\% iso-concentration surface. Iso-concentration surfaces overlaid on a $2 \mathrm{D}$ contour plot with the stand-alone $2 \mathrm{D}$ contour plot shown in a larger view, for (c) Fe and (d) Ni. Proximity histograms across (e) 54 at.\% Fe and (f) 32 at.\% Ni iso-concentration surface. (g) Compositions of volumes encapsulated by 54 at.\% Fe, 32 at.\% Ni, and 12 at.\% B iso-concentration surface with estimated volume percent of each region identified in parentheses. The total volume analyzed was $3.0295 \times 10^{4} \mathrm{~nm}^{3}$.

enriched crystalline regions are also found to be depleted in $\mathrm{B}$, $\mathrm{Si}$, and $\mathrm{Nb}$. This observation suggests that $\mathrm{B}, \mathrm{Si}$, and $\mathrm{Nb}$ may encompass the $\mathrm{Fe}$ and Ni-rich crystalline regions which are found to be in direct contact with each other via HRTEM (Fig. 2). By investigating sub-volumes of the annealed data set via $\mathrm{APT}$, we find adjacent $\mathrm{Fe}$ and Ni-rich regions in direct contact with each other, consistent with Fig. 2 HRTEM results. This observation is shown in Figs. 5(a) and 5(b) 2D contour plots, where $\mathrm{Fe}$ and $\mathrm{Ni}$ rich regions are highlighted with a dashed line. A corresponding $\mathrm{Nb}$ enrichment on the periphery of these adjacent $\mathrm{Fe}$ and $\mathrm{Ni}$-enriched regions can also be seen in the Fig. 5(c) Nb contour plot. The $\mathrm{Nb}$ enrichment is indicated by several black arrows and appears to be a discontinuous shell around the $\mathrm{Fe}$ and Ni nanocrystals. Corresponding concentration profiles for $\mathrm{Fe}, \mathrm{Ni}$, and $\mathrm{Nb}$ [Figs. 5(d)-5(f)] indicate $\mathrm{Fe}$ and $\mathrm{Ni}$ depleted regions correspond to the $\mathrm{Nb}$ enrichment above the 1.07 at.\% average in the entire reconstructed volume. The $\mathrm{Nb}$ enrichment above the average 1.065 at.\% is labeled as the $\mathrm{Nb}$-shell in Fig. 5(f) and is approximately $4 \mathrm{~nm}$ wide in the sub-volume analyzed here. The $\mathrm{Nb}$ enrichment shown in Figs. 5(c) and 5(f) can also be correlated with an increase in $\mathrm{B}$ and $\mathrm{Si}$ concentration. It is interesting to note that the location of the $\mathrm{Nb}$ enrichment on the periphery of the Fe and Ni rich nanocrystals is consistent with previous discussions of an $\mathrm{Nb}$-shell which tends to surround the growing nanocrystals and acts to inhibit further crystalline growth [26].

To illustrate and explain the tendency for Fe-segregation to the BCC nanocrystalline phase and Ni-segregation to the FCC nanocrystalline phase, Gibbs free energy versus composition curves were extracted from Thermocalc and plotted at the crystallization temperature for binary FeNi alloys in the BCC, FCC, and liquid phase as well as for more realistic compositions in the liquid phase. Free energy curves are presented in Fig. 6 as well as tangent lines which help to indicate the BCC and FCC compositions for a nominal binary alloy composition of $\mathrm{Fe}_{0.7} \mathrm{Ni}_{0.3}$ which satisfy both the (i) common tangency condition corresponding to phase equilibrium (circles) and (ii) parallel tangency condition corresponding to the composition of each phase with the highest probability to nucleate from the liquid (arrows) [14]. It can be observed that for both conditions, Ni-enrichment of the FCC phase and Fe-enrichment of the BCC phase would be expected, and the predictions for both are similar in terms of the compositions identified. While the predicted enrichment of $\mathrm{Ni}$ in the FCC phase is 


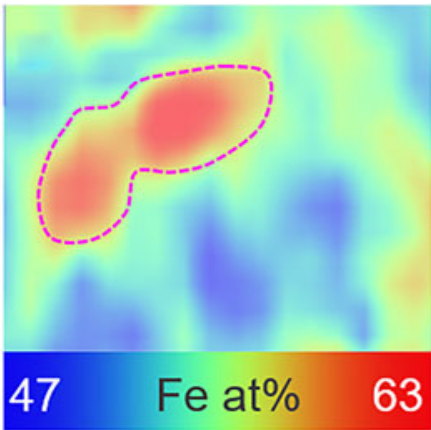

(a)

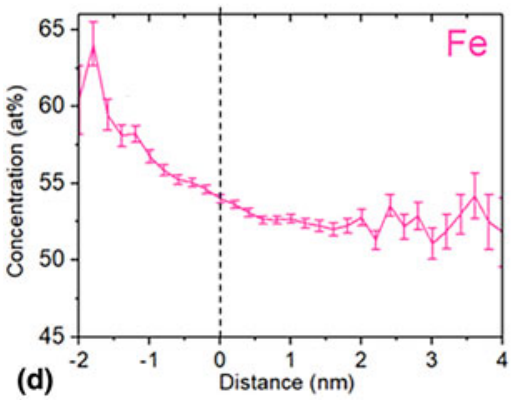

(b)
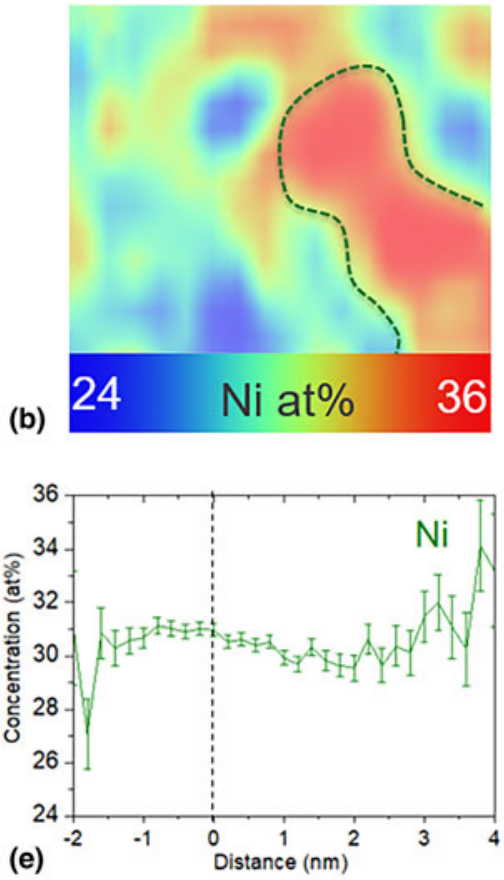

(c)
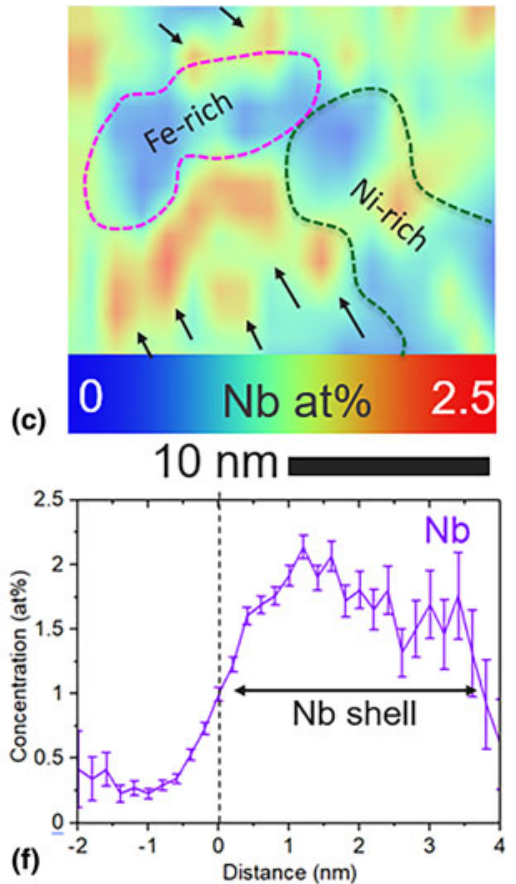

Figure 5: Elemental segregation in a sub-volume of the annealed alloy analyzed via APT. 2D contour plots for (a) Fe, (b) Ni, and (c) Nb are provided with Fe and Ni-rich regions outlined in pink and green, respectively. In (c), black arrows point toward regions of Nb enrichment at the surface of crystalline regions. Corresponding concentration profiles for $\mathrm{Fe}, \mathrm{Ni}$, and $\mathrm{Nb}$ are given in $(\mathrm{d}-\mathrm{f})$, respectively for a $1 \mathrm{at} . \% \mathrm{Nb}$ iso-concentration surface. The vertical dashed lines in the concentration profiles indicate the location of the 1 at.\% Nb iso-concentration surface. 2D contour plots were generated from a volume of $18 \mathrm{~nm} \times 19 \mathrm{~nm} \times 1 \mathrm{~nm}$. Concentration of each element was analyzed in the thickness direction. Color scale bars provide the concentration of the selected element in at. \%.
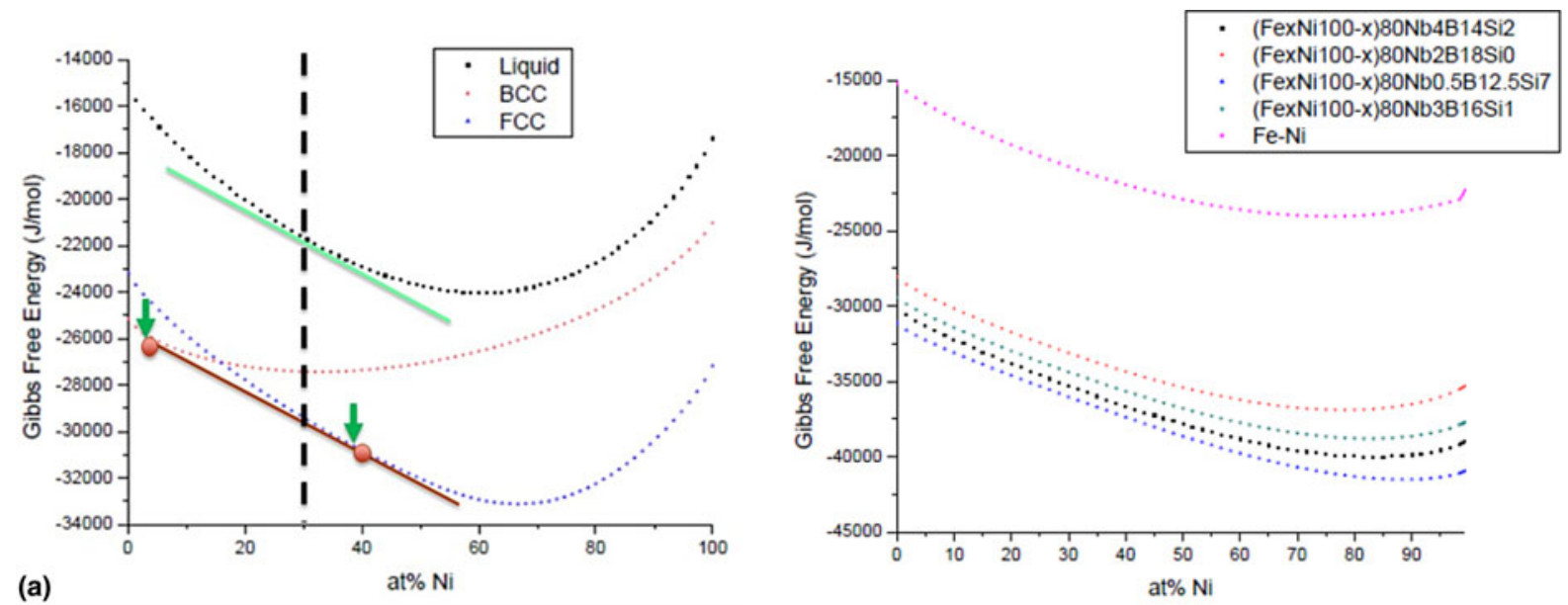

Figure 6: (a) Free energy versus composition curves for BCC, FCC, and liquid binary FeNi alloy at $440{ }^{\circ} \mathrm{C}$ (crystallization temperature) and (b) corresponding free energy versus composition curves for realistic liquid phase compositions. (Green arrows/line = parallel tangency condition to maximize driving force for nucleation, red circles/line = common tangency condition for equilibrium, following the methods described previously [14]).

reasonably consistent with the experimentally determined compositions of the $\mathrm{Ni}$-enriched regions from a quantitative perspective ( $\sim 38-40 \% \mathrm{Ni}$ predicted, $\sim 42 \%$ experimentally) that of $\mathrm{Fe}$ in the BCC phase is greatly overestimated ( $95-$ $97 \%$ Fe predicted, $\sim 68 \%$ experimentally) by the predictions. In the case of Co-rich alloys, more realistic thermodynamic predictions for the amorphous phase by incorporating experimentally derived enthalpies of crystallization and/or firstprinciples calculations improved quantitative agreement using the parallel tangency condition [14]. Such improvements may also be needed in the case of the FeNi alloys as well but are beyond the scope of the current study. As a first approximation, free energy versus composition curves of more realistic liquid phase chemistries were extracted from Thermocalc as presented 
in Fig. 6(b) and compared with the binary FeNi-liquid phase. Similar slopes of all free energy curves across the composition range of interest surrounding Fe:Ni ratios of 70:30 would not explain the deviation between experiment and theory for the Fe-enriched BCC regions. In contrast, kinetic limitations may preclude compositional segregation of Fe to such a large extent during the crystallization process. More rigorous analysis of the thermodynamic driving forces for compositional segregation may also be required and can be the subject of future research and investigations.

\section{Conclusions}

A detailed, nanoscale experimental investigation of a $\left(\mathrm{Fe}_{70} \mathrm{Ni}_{30}\right)_{80} \mathrm{Nb}_{4} \mathrm{Si}_{2} \mathrm{~B}_{14}$ MANC alloy with multiple nanocrystalline phases is presented using a combination of TEM and APT to explore both microstructure and compositional segregation at the nanoscale. HRTEM confirms that both individual BCC and FCC nanocrystals are embedded within an amorphous matrix, and BCC and FCC nanocrystals are in direct contact with one other exhibiting well-known BCC/FCC orientation relationships. Complementary APT results confirm compositional segregation associated with the crystallization process which occurred after casting and annealing. Results also show evidence for three distinct types of regions within the microstructure: (i) Fe-enriched, (ii) Ni-enriched, and (iii) B-enriched regions which are attributed to the BCC, FCC, and amorphous intergranular phases, respectively. Evidence for $\mathrm{Nb}$ enrichment (i.e., the "Nb-shell") surrounding crystalline regions is also experimentally observed via APT. The Nb-shell is hypothesized to play an important role in limiting the characteristic microstructural feature size to the nm-range for MANCs by acting as a diffusion barrier which prevents grain coarsening. The application of both the common and parallel tangency condition to describe BCC and FCC composition of the thermodynamically stable and highest thermodynamic driving force, respectively, show similar results and a tendency for Fe-enrichment of BCC and Ni-enrichment of FCC phases, further confirming experimental results. Results presented here provide an enhanced understanding of the nanoscale structural and compositional evolution of emerging alloys for power and energy applications spanning electric motors, transformers, and others.

\section{Materials and Methods}

Bulk alloy ingots of composition $\left(\mathrm{Fe}_{70} \mathrm{Ni}_{30}\right)_{80} \mathrm{Nb}_{4} \mathrm{Si}_{2} \mathrm{~B}_{14}$ were prepared from constituent elemental materials through arc melting techniques and used as feedstock for rapid solidification processing with an Edmund Buehler MSP 10, employing a $\mathrm{Cu}$ wheel with a surface speed of $35 \mathrm{~m} / \mathrm{s}$. Amorphous metal ribbons were confirmed after rapid solidification processing through $\mathrm{X}$-ray diffraction and bend-tests which showed excellent ductility. Ribbons were then wound into a toroidal core and subjected to an annealing treatment of $T=$ $440{ }^{\circ} \mathrm{C}$ for $1 \mathrm{~h}$ to produce a partially devitrified MANC alloy. The temperature was selected to produce optimized magnetic properties as reported in prior work [3,4]. A sample was prepared for TEM analysis through standard lift-out techniques using a NOVA Focused Ion Beam (FIB) based scanning electron microscope (SEM). Samples were approximately $10 \mu \mathrm{m}$ in width and 5-10 $\mu \mathrm{m}$ in depth after the final thinning stages to successfully realize electron transparency. High-resolution and high-angle annular dark-field imaging were then performed using both an FEI Tecnai F20 and an aberrationcorrected FEI Titan G2 80-300 TEM/STEM from a region farther than $500 \mathrm{~nm}$ from the top surface to ensure a microstructure representative of the ribbon bulk.

Needle specimens for APT analysis were prepared from $\mathrm{Fe}-$ $\mathrm{Ni}$ as cast and annealed ribbons. Bulk FIB lift-outs were performed to shape APT needles from as-cast and annealed samples. A CAMECA LEAP 4000× HR system was used for data collection in the voltage pulsing mode. User-selected parameters for data collection included: $200 \mathrm{kHz}$ pulse repetition rate, $20 \%$ pulse fraction, $45 \mathrm{~K}$ specimen temperature, and 0.005 atoms/pulse detection rate. Data reconstruction and analysis was performed using IVAS version 3.8.2.

Gibbs free energy curves were generated using Thermocalc 2018 b using the TCFE9 Steel/Fe-Alloys v9.0 database. Gibbs free energy was plotted for the crystallization products, and the liquid as $\mathrm{Ni}$ content was varied from 0 to $100 \%$ in an Fe-Ni binary alloy. The Gibbs free energy curves were also plotted versus \%Ni for the liquid phase in pseudo-binary alloys containing different glass former compositions.

\section{Acknowledgments}

APT sample preparation and analysis was conducted using facilities at the Environmental Molecular Sciences Laboratory, a national scientific user facility sponsored by the DOE's Office of Biological and Environmental Research and located at the Pacific Northwest National Laboratory (PNNL). PNNL is operated for the U.S. DOE by Battelle Memorial Institute under contract no. DE-AC05-76RLO1830).

\section{References}

1. A.M. Leary, P.R. Ohodnicki, and M.E. McHenry: Soft magnetic materials in high-frequency, high-power conversion applications. JOM 64, 772 (2012).

2. Y. Yoshizawa, S. Oguma, and K. Yamauchi: New Fe-based soft magnetic alloys composed of ultrafine grain structure. J. Appl. Phys. 64, 6044-6046 (1988). 
3. N. Aronhime, E. Zoghlin, V. Keylin, X. Jin, P. Ohodnicki, and M.E. McHenry: Magnetic properties and crystallization kinetics of $\left(\mathrm{Fe}_{100-\mathrm{x}} \mathrm{Ni}_{\mathrm{x}}\right)_{80} \mathrm{Nb}_{4} \mathrm{Si}_{2} \mathrm{~B}_{14}$ metal amorphous nanocomposites. Scr. Mater. 142, 133-137 (2018).

4. N. Aronhime, V. DeGeorge, V. Keylin, P. Ohodnicki, and M.E. McHenry: The effects of strain-annealing on tuning permeability and lowering losses in Fe-Ni based metal amorphous nanocomposites. JOM 69, 2164-2170 (2017).

5. J.I. Ipus, P. Herre, P. Ohodnicki, and M.E. McHenry: High temperature $\mathrm{x}$-ray diffraction determination of the body-centered-cubic-face-centered-cubic transformation temperature in $\left(\mathrm{Fe}_{70} \mathrm{Ni}_{30}\right)_{88} \mathrm{Zr}_{7} \mathrm{~B}_{4} \mathrm{Cu}_{1}$ nanocomposites. J. Appl. Phys. 111, 07 A323 (2012).

6. P.R. Ohodnicki Jr., S.Y. Park, D.E. Laughlin, M.E. McHenry, V. Keylin, and M.A. Willard: Crystallization and thermomagnetic treatment of a Co-rich $\mathrm{Co}-\mathrm{Fe}-\mathrm{Ni}-\mathrm{Zr}-\mathrm{B}-\mathrm{Cu}$ based nanocomposite alloy. J. Appl. Phys. 103, 07E729 (2008).

7. S.J. Kernion, P.R. Ohodnicki, and M.E. McHenry: In-situ investigation of phase formation in nanocrystalline $\left(\mathrm{Co}_{97.5} \mathrm{Fe}_{2.5}\right)_{89} \mathrm{Zr}_{7} \mathrm{~B}_{4}$ alloy by high temperature $\mathrm{x}$-ray diffraction. J. Appl. Phys. 111, 07A316 (2012).

8. P.R. Ohodnicki, J. Long, D.E. Laughlin, M.E. McHenry,

V. Keylin, and J. Huth: Composition dependence of field induced anisotropy in ferromagnetic $(\mathrm{Co}, \mathrm{Fe}){ }_{89} \mathrm{Zr}_{7} \mathrm{~B}_{4}$ and (Co, $\mathrm{Fe})_{88} \mathrm{Zr}_{7} \mathrm{~B}_{4} \mathrm{Cu}_{1}$ amorphous and nanocrystalline ribbons. J. Appl. Phys. 104, 113909 (2008).

9. R. Goswami and M.A. Willard: Microstructure evolution in rapidly solidified ferromagnetic $\left(\mathrm{Co}_{0.95} \mathrm{Fe}_{0.05}\right)_{89} \mathrm{Zr}_{7} \mathrm{~B}_{4}$ nanocrystalline alloys. Scr. Mater. 59, 459-462 (2008).

10. M. Daniil, P.R. Ohodnicki, M.E. McHenry, and M.A. Willard: Shear band formation and fracture behavior of nanocrystalline (Co,Fe)-based alloys. Philos. Mag. 90, 1547-1565 (2009).

11. S.J. Kernion, P.R. Ohodnicki, J. Grossman, A. Leary, S. Shen, V. Keylin, J.F. Huth, J. Horwath, M.S. Lucas, and

M.E. McHenry: Giant induced magnetic anisotropy in strain annealed Co-based nanocomposite alloys. Appl. Phys. Lett. 101, 102408 (2012).

12. Y. Krimer, V. Keylin, A.M. Leary, P.R. Ohodnicki, K. Byerly, A. Wise, E.A. Clark, and M.E. McHenry: Mechanical properties of strain annealed metal amorphous nanocomposite (MANC) soft magnetic material. Materialia 4, 323-330 (2018).

13. A.M. Leary, V. Keylin, P. R. Ohodnicki, and M.E. McHenry: Stress induced anisotropy in CoFeMn soft magnetic nanocomposites. J. Appl. Phys. 117, 17A338 (2015).

14. P.R. Ohodnicki, D.E. Laughlin, M.E. McHenry, and M. Widom: Application of classical nucleation theory to phase selection and composition of nucleated nanocrystals during crystallization of
Co-rich (Co,Fe)-based amorphous precursors. Acta Mater. 58, 4804-4813 (2010).

15. P.R. Ohodnicki, Y.L. Qin, D.E. Laughlin, M.E. McHenry, M. Kodzuka, T. Ohkubo, K. Hono, and M.A. Willard: Composition and non-equilibrium crystallization in partially devitrified co-rich soft magnetic nanocomposite alloys. Acta Mater. 57, 87-96 (2009).

16. P.R. Ohodnicki, Y. Qin, M.E. McHenry, D.E. Laughlin, and V. Keylin: Transmission electron microscopy study of large field induced anisotropy $\left(\mathrm{Co}_{1-\mathrm{x}} \mathrm{Fe}_{\mathrm{x}}\right) \mathrm{Zr89Zr7B4}$ nanocomposite ribbons with dilute Fe-contents. J. Magn. Magn. Mater. 322, 315-321 (2010).

17. P.R. Ohodnicki, S.Y. Park, H.K. McWilliams, K. Ramos, D.E. Laughlin, and M.E. McHenry: Phase evolution during crystallization of nanocomposite alloys with Co:Fe ratios in the two-phase region of the binary Fe-Co phase diagram. J. Appl. Phys. 101, 09N108 (2007).

18. F. Barcelo, J.L. Bechade, and B. Fournier: Orientation relationship in various $9 \% \mathrm{Cr}$ ferritic/martensitic steels-EBSD comparison between Nishiyama-Wassermann, Kurdjumov-Sachs and Greninger-Troiano. Phase Trans. 83, 601-614 (2010).

19. Z. Nishiyama: Martensitic Transformation (Elsevier Science, New York, 1978).

20. G. Wassermann: Uber den Mechanismus der alpha-gamma Umwandlung des Eisens. Mitteilungen aus dem Kaiser-Wilhelm-Institut fur Eisenforschung zu Dusseldorf (Stahleisen, Verlag, Dusseldorf, Germany, 1935).

21. G.V. Kurdjumov and G. Sachs: Uber den Mechanismus der Stahlhartung. Zeitschrift fur Physik 64, 325-343 (1930).

22. B. Gault, M.P. Moody, J. M. Cairney, and S.P. Ringer: Atom Probe Microscopy, Vol. 160 (Springer Science \& Business Media, 2012).

23. M.P. Moody, L.T. Stephenson, P.V. Liddicoat, and S.P. Ringer: Contingency table techniques for three dimensional atom probe tomography. Microsc. Res. Techniq. 70, 258-268 (2007).

24. M.P. Moody, L.T. Stephenson, A.V. Ceguerra, and S.P. Ringer: Quantitative binomial distribution analyses of nanoscale likesolute atom clustering and segregation in atom probe tomography data. Microsc. Res. Techniq. 71, 542-550 (2008).

25. O.C. Hellman, J. Vandenbroucke, J. Rusing, D. Isheim, and D.N. Seidman:Analysis of three-dimensional atom-probe data by the proximity histogram. Microsc. Microanal. 6, 437-444 (2000).

26. S.J. Kernion, V. Keylin, J. Huth, and M.E. McHenry: Secondary crystallization in $\left(\mathrm{Fe}_{65} \mathrm{Co}_{35}\right)_{79.5+\mathrm{x}} \mathrm{B}_{13} \mathrm{Nb}_{4-\mathrm{x}} \mathrm{Si}_{2} \mathrm{Cu}_{1.5}$ and $\left(\mathrm{Fe}_{65} \mathrm{Co}_{35}\right)_{83} \mathrm{~B}_{10} \mathrm{Nb}_{4} \mathrm{Si}_{2} \mathrm{Cu}_{1}$ nanocomposite alloys. J. Appl. Phys. 111, 07A329 (2012). 